\title{
Management von Data Science
}

\section{Liebe Leserinnen und Leser,}

Thomas H. Davenport und DJ Patil haben 2012 in einem häufig zitierten Beitrag in der „Harvard Business Review“ das Berufsbild des Data Scientists als „The Sexiest Job of the 21st Century“ tituliert. Aber eine schöne neue Welt kommt nicht von allein, sondern muss mit Blick auf ihre Chancen und Risiken aktiv gestaltet werden. Beginnen wir mit den Chancen: Data Science erschließt nicht nur gänzlich neue Datenwelten. Sie ermöglicht auch den Blick auf das einzelne Daten-Item und somit einen Bruch im Denken, weg von Durchschnitten, hin zum Einzelfall. Standardinstrumente des Controllings wie die operative Planung oder die Kostenrechnung sind von einem Durchschnittsdenken beherrscht. Data Science lässt nun Feinstrukturen und Muster hervortreten und eröffnet damit ein immenses, bislang ungenutztes Analyse- und Handlungspotenzial. Daneben erhöht sie die analytische Freiheit. Auswertungen sind nicht mehr an Kalenderzeitperioden („Monatsbericht“) oder feste Auswertungsschemata gebunden.

All diesen Chancen stehen aber auch Risiken gegenüber. Schnell wird Data Science überhöht, ihre Resultate einer kritischen Diskussion entzogen. Je „besser“ die Daten der Vergangenheit ausgewertet werden, desto „richtiger“ sehen sie aus. Systematische Verzerrungen in der Datenbasis werden aber durch den besten Algorithmus nicht geheilt. Und alle wirklich neuen Themen werden von auf Vergangenheitsdaten aufbauenden Algorithmen grundsätzlich nicht erfasst. Rationalitätssicherung - nicht zuletzt durch Controller - ist somit wichtiger denn je. Dies betrifft auch den Umgang mit Algorithmen. Hier gilt es, nicht von der Methodik her zu kommen, sondern von der Frage und vom Problem. Um aber die richtigen Fragen stellen zu können, bedarf es eines fundierten Verständnisses des zugrunde liegenden Geschäfts. Damit ist in erster Linie das Management gefragt. Und für Controller naht der Moment der Wahrheit: Sind sie gut genug aufgestellt, um durch ihre Geschäftskenntnisse, ihre Toolbox und durch einen neutralen Drittblick adäquat zu unterstützen? Wenn ja, kann ihnen in der neuen Welt eine ganz zentrale Rolle zukommen. Diese wird man ihnen aber nur selten kampflos überlassen. Entsprechend gilt es, das Zusammenspiel von Management, Data Scientists und Controllern bewusst zu gestalten. Data Science der Linie

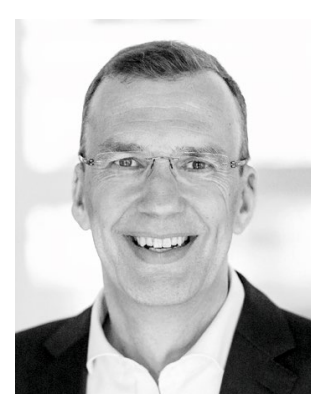

Utz Schäffer

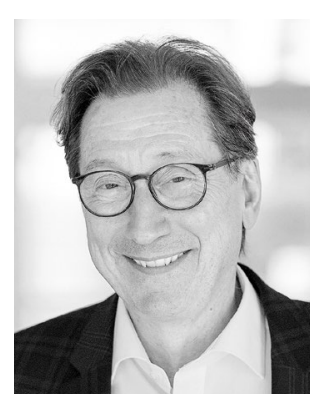

Jürgen Weber oder Funktionen wie Marketing oder Supply Chain zuzuordnen, sichert Geschäfts-Know-how, lässt aber keinen übergreifenden Erfahrungsgewinn zu. Die Federführung in einer speziellen Data-Science-Abteilung fokussiert stark auf die Methodik. Das Controlling in die Verantwortung zu nehmen, fördert schließlich eine Gesamtperspektive und erleichtert die Verknüpfung von finanziellen und nichtfinanziellen Datenpunkten, setzt aber voraus, dass Controller nahe genug am Geschäft sind und es genügend Fragestellungen gibt, die einen problembeziehungsweise bereichsübergreifenden Charakter haben.

In der Praxis finden sich all die genannten Lösungen, aber kaum eine tiefer gehende Auseinandersetzung mit der Organisationsfrage. Vielmehr werden vielfach frühe Weichenstellungen und Startvorteile fortgeschrieben, oder Machtfragen und persönliche Initiative dominieren das fachliche Argument. Aber (beinahe) unabhängig davon, wie die Entscheidung am Ende ausfällt: Sie nicht zu treffen, verzögert die Fahrt in die schöne neue Welt. Und dies wird man sich vielfach nicht erlauben können, denn der Kampf um die besten Plätze im digitalen Wettbewerb hat längst begonnen.

Viel Spaß bei der Lektüre wünschen Ihnen
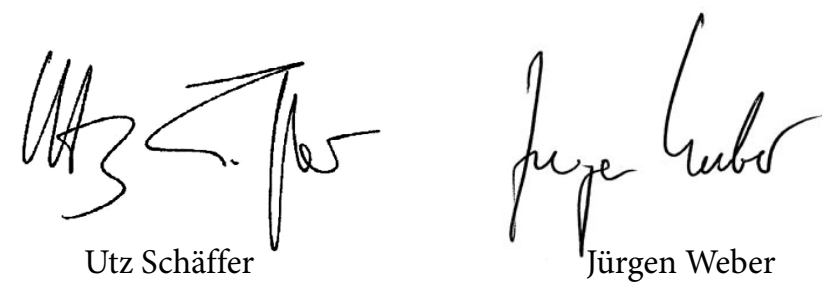